\title{
Hair Metabolomics: Identification of Fetal Compromise Provides Proof of Concept for Biomarker Discovery
}

\author{
Karolina Sulek ${ }^{1,2}{ }^{\bowtie *}$, Ting-Li Han ${ }^{1,2^{*}}$, Silas Granato Villas-Boas², David Scott Wishart ${ }^{3}$, Shu-E Soh ${ }^{4,5}$, Kenneth \\ Kwek $^{6}$, Peter David Gluckman ${ }^{1,7}$, Yap-Seng Chong7,8, Louise Claire Kenny ${ }^{9}$, Philip Newton Baker ${ }^{1}$ \\ 1. The Liggins Institute, University of Auckland, Auckland, New Zealand \\ 2. School of Biological Sciences, University of Auckland, Auckland, New Zealand \\ 3. Departments of Biological Sciences and Computing Science, University of Alberta, Edmonton, Canada \\ 4. Saw Swee Hock School of Public Health, National University of Singapore, National University Health System, Singapore \\ 5. Department of Paediatrics, Yong Loo Lin School of Medicine, National University of Singapore, National University Health System, \\ Singapore \\ 6. Department of Maternal and Fetal Medicine, KK Women's and Children's' Hospital, Singapore \\ 7. Singapore Institute for Clinical Sciences, Agency for Science, Technology and Research, Singapore \\ 8. Department of Obstetrics \& Gynaecology, Yong Loo Lin School of Medicine, National University of Singapore, National University \\ Health System, Singapore \\ 9. The Irish Centre for Fetal and Neonatal Translational Research, University College Cork, Cork, Ireland \\ * Authors contributed equally.
}

$\square$ Corresponding author: Karolina Sulek; Private Bag 92019, Auckland 1142, New Zealand; T: +64 99234751 ; F: +64 9 373 7039 ; k.sulek@auckland.ac.nz.

() Ivyspring International Publisher. This is an open-access article distributed under the terms of the Creative Commons License (http://creativecommons.org/ licenses/by-nc-nd/3.0/). Reproduction is permitted for personal, noncommercial use, provided that the article is in whole, unmodified, and properly cited.

Received: 2014.03.3I; Accepted: 2014.06.12; Published: 2014.07.20

\begin{abstract}
Analysis of the human metabolome has yielded valuable insights into health, disease and toxicity. However, the metabolic profile of complex biological fluids such as blood is highly dynamic and this has limited the discovery of robust biomarkers. Hair grows relatively slowly, and both endogenous compounds and environmental exposures are incorporated from blood into hair during growth, which reflects the average chemical composition over several months.

We used hair samples to study the metabolite profiles of women with pregnancies complicated by fetal growth restriction (FGR) and healthy matched controls. We report the use of GC-MS metabolite profiling of hair samples for biomarker discovery. Unsupervised statistical analysis showed complete discrimination of FGR from controls based on hair composition alone. A predictive model combining 5 metabolites produced an area under the receiver-operating curve of 0.998 .

This is the first study of the metabolome of human hair and demonstrates that this biological material contains robust biomarkers, which may lead to the development of a sensitive diagnostic tool for FGR, and perhaps more importantly, to stable biomarkers for a range of other diseases.
\end{abstract}

Key words: metabolite profiling; hair; biomarker; fetal growth restriction; GC-MS.

\section{Introduction}

Metabolic profiling is a powerful strategy for investigating the low molecular weight metabolites that represent the metabolome of a cell, tissue, or organism ${ }^{1}$. The metabolome's position as a downstream product of gene expression enables the provision of a high-resolution multifactorial phenotypic signature of disease etiology, manifestation, or pathophysio$\log \mathrm{y}^{2,3,4}$.

The search for biomarker candidates in biological samples can be attempted by using the appropri- 
ate analytical tools 5 . However, the metabolome of complex biological fluids such as blood and urine is highly dynamic, containing a significant variety and concentration of metabolites; the subsequent low signal to noise ratio has limited the discovery of robust biomarkers ${ }^{6,7,8}$. Analysis of blood requires invasive sampling, immediate processing and curation of samples under controlled circumstances if analysis is not immediate. In addition, the metabolome of blood is highly dynamic as it changes on a minute-to-minute basis and it is highly dependent on diet and immune status. Hair, on the other hand, offers several potential advantages over blood for metabolite profiling. It is a highly stable structure retaining endogenous compounds and reflecting environmental exposures for many months ${ }^{9}$. Secondly, hair sampling is non-invasive and the storage and processing of hair is much simpler and more efficient than blood, making it a particularly useful source of biomarkers in low resource settings.

Fetal Growth Restriction (FGR) complicates 4-7\% of pregnancies worldwide and occurs when babies do not reach their in utero growth potential. FGR is most commonly defined using the surrogate of small for gestational age (SGA) (fetal weight below the $10^{\text {th }}$ percentile for gestational age). FGR confers increased risks of perinatal morbidity and mortality ${ }^{10,11}$ and increases the risk of cardiovascular complications and diabetes in later life ${ }^{13}$. Early recognition of FGR facilitates increased fetal surveillance and leads to a reduction in perinatal mortality. In addition, preventative strategies with treatments such as low dose aspirin depend on accurate prediction of the 'at risk' fetus ${ }^{12}$

Current strategies for the prediction and the detection of FGR depend upon screening by measurement of the symphysis-fundal height (SFH), followed by biometric and Doppler measurements of the fetus using high-resolution ultrasound ${ }^{13}$. However, measurement of the SFH has both low sensitivity and specificity and only detects $16 \%$ of FGR fetuses ${ }^{14}$. Consequently, there is a need for better assays and more robust biomarkers for early detection ${ }^{15}$.

\section{Methods}

\section{Ethical Statement}

Studies were approved by the Auckland District Health Board and the Health and Disability Ethics Committees ref. 13/NTA/7. In Singapore, ethical approval for the study was granted, by the ethics boards of both KK Women's and Children's Hospital (KKH) and the National University Hospital (NUH), which are the Centralised Institute Review Board (CIRB) and Domain Specific Review Board (DSRB), respectively.

\section{Chemicals and Reagents}

Chloroform, methanol, potassium hydroxide, sodium bicarbonate, sodium hydroxide were purchased from MERK (Damstadt, Germany). The internal standard 2,3,3,3-d4-alanine, the derivatization reagent methylchloroformate $(\mathrm{MCF})$, and pyridine were obtained from Sigma-Aldrich (St. Louis, USA). Anhydrous sodium sulphate and were obtained from Fluka (Steinheim, Germany). Sulphuric acid was brought from ROMIL Pure Chemistry (Lameira, England). All chemicals used in this research were of analytical grade.

\section{Study Participants}

Women were recruited from the prospective, longitudinal Growing $\underline{\mathrm{U}} \mathrm{p}$ in Singapore Towards healthy Outcomes (GUSTO) cohort study which has been described in detail elsewhere ${ }^{16,17}$. 41 women who subsequently were delivered of an fetus (defined as SGA less than the $10^{\text {th }}$ corrected birth centile) were matched for maternal age, BMI at antenatal booking, ethnicity and the gender of the fetus with 42 women who subsequently delivered an appropriately grown fetus.

\section{Hair Samples}

Hair samples were collected at 26-28 weeks of gestation. Hairs were cut around the occipital area, $0.5 \mathrm{~cm}$ away from the scalp with blunt scissors. Samples were stored at $-20^{\circ} \mathrm{C}$ until further use. Samples below $1.5 \mathrm{mg}$ in weight were disregarded.

\section{Sample Preparation}

All hair samples were washed in MQ water and methanol three times. $1.5 \mathrm{mg}-5.5 \mathrm{mg}$ of hair (2-3 strands) were cut into $5 \mathrm{~mm}$ segments and spiked with $0.2 \mu \mathrm{mol}$ of $2,3,3,3-\mathrm{d}_{4}$-alanine. Hair samples were incubated in $1 \mathrm{~mL}$ of $1 \mathrm{M}$ potassium hydroxide at $54^{\circ} \mathrm{C}$ for $24 \mathrm{~h}$. After alkaline hydrolysis, the hair extracts were neutralised to $\mathrm{pH} 7$ by addition of sulphuric acid and evaporated by freeze drying (BenchTop K Manifold Freeze Dryer, VirTis). To remove salts and other residues, lyophilised samples were resuspended in $1 \mathrm{~mL}$ of methanol water $(70 \% \mathrm{v} / \mathrm{v})$ and spun in a centrifuge at $2000 \mathrm{~g}$ for $5 \mathrm{~min}$ to separate the supernatant from the insoluble hair debris. The supernatants containing hair extracts were allowed to evaporate to dryness using a rotary evaporator (Savant SPS121P Speedvac, Thermo) at $37^{\circ} \mathrm{C}$ for $12 \mathrm{~h}$ before derivatization. Samples were chemically derivatised using a methyl chloroformate (MCF) method in accordance with the protocol published by Smart $e t$ al. ${ }^{18}$ In summary, samples were resuspended in $200 \mu \mathrm{L}$ $1 \mathrm{M}$ sodium hydroxide and transferred to a silanised glass tube. $167 \mu \mathrm{L}$ methanol and $34 \mu \mathrm{L}$ pyridine were 
then added to the solution. The derivatization was initiated by adding $20 \mu \mathrm{L}$ MCF followed by vigorous mixing for 30s, and then a further $20 \mu \mathrm{L}$ MCF was added accompanied by another 30 s of vigorous mixing. To separate the MCF derivatives, $400 \mu \mathrm{L}$ chloroform was added, mixed for 10 s and then $400 \mu \mathrm{L}$ of $50 \mathrm{mM}$ sodium bicarbonate was added and mixed for an additional 10s. The aqueous layer was discarded and dehydrated with anhydrous sodium sulphate before samples were transferred to GC vials for GC-MS analysis.

\section{GC-MS analysis}

The MCF derivatives were analyzed in a Thermo Trace GC Ultra system coupled to an ISQ mass selective detector (EI) setting at $70 \mathrm{eV}$. The gas column equipped for all analyses was a ZB-1701 GC capillary column $(30 \mathrm{~m} \times 250 \mu \mathrm{m}$ id $\times 0.15 \mu \mathrm{m}$ with $5 \mathrm{~m}$ guard column, Phenomenex). The analysis parameters were conducted according to Smart et al. ${ }^{18}$ Samples were injected under pulsed splitless mode with the injector temperature at $260^{\circ} \mathrm{C}$. The helium gas flow through the GC-column was set at a constant flow of $1 \mathrm{~mL}$ $\mathrm{min}^{-1}$. The GC-oven temperature was initially held at $45^{\circ} \mathrm{C}$ for $2 \mathrm{~min}$, and then raised with a gradient of $9^{\circ} \mathrm{C} \cdot \mathrm{min}^{-1}$ to $180^{\circ} \mathrm{C}$; after $5 \mathrm{~min}$. the temperature ramped at $40^{\circ} \mathrm{C} \cdot \mathrm{min}^{-1}$ to $220^{\circ} \mathrm{C}$. After a further $5 \mathrm{~min}$, the temperature was ramped at $40^{\circ} \mathrm{C} \cdot \mathrm{min}^{-1}$ to $240^{\circ} \mathrm{C}$ for $11.5 \mathrm{~min}$; finally, the temperature was ramped at $40^{\circ} \mathrm{C}$. $\mathrm{min}^{-1}$ until it reached $280^{\circ} \mathrm{C}$ and held for at $280^{\circ} \mathrm{C}$ for $2 \mathrm{~min}$. The interface temperature was set to $250^{\circ} \mathrm{C}$ and the quadrupole temperature was $230^{\circ} \mathrm{C}$. The mass spectrometry was operated in scan mode and started after 5.5min with mass range between 38-550 amu with scan time of $0.1 \mathrm{~s}$.

\section{Statistical Analysis}

AMDIS software (version 2.66) was used for deconvoluting GC-MS chromatograms and identifying metabolites through the use of our in-house MCF mass spectra library. Compound identifications were based on matches to both the MS spectrum of the derivatised metabolite and its respective chromatographic retention time. The relative abundance of identified metabolites was determined by Xcalibur (Thermo) by integration and using the GC base-peak value of a specific reference ion. These values were normalised by the hair weight in each sample as well as by the abundance of internal standard (2,3,3,3-d $\mathrm{d}_{4}$-alanine). Raw chromatographic data mining and data normalisation were automated by $\mathrm{R}$ Metab package as described in Aggio et al. ${ }^{19}$. The differences in the relative abundance of each identified metabolite between experimental conditions were compared and validated through an analysis of
Wilcoxon-Mann-Whitney test and multivariate analysis, using MetaboAnalyst 2.0 20. Multivariate Receiver Operating Characteristic (ROC) curves were calculated using the ROCCET (ROC Curve Explorer and Tester) web server ${ }^{21}$. Chromatographic data was log-transformed and autoscaled prior to all statistical modelling. Graphical representations of the metabolites ratios between groups (Figure 1) were produced by the ggplot2 $\mathrm{R}$ packages ${ }^{22}$. Statistical analysis of the clinical (population) data was performed using unpaired Student T-test with Welch's correction.

\section{Results and Discussion}

Hair samples obtained at 26-28 weeks' gestation from 41 women participating in the GUSTO study ${ }^{26}$ who subsequently delivered FGR babies $\left(<10^{\text {th }}\right.$ centile) and 42 controls who delivered appropriately grown babies after 37 weeks of gestation were subject to hair metabolite profiling using GC-MS. The clinical characteristics of the women and their babies are detailed in Table 1. All women were assessed as 'low risk' at the start of the pregnancy. There were no statistical differences between the two groups in maternal age, ethnicity, parity, BMI (determined at the antenatal booking visit) or gestational age at delivery. By definition, babies born FGR had significantly lower crude birth weights and lower birth weight for gestational age centiles.

Table I. Demographic and clinical characteristics of the study population.

\begin{tabular}{|c|c|c|}
\hline Characteristic & FGR Case $(n=41)$ & Control $(n=42)$ \\
\hline \multirow[t]{3}{*}{ Ethnicity } & 23 Chinese & 21 Chinese \\
\hline & 8 Indian & 9 Indian \\
\hline & 10 Malay & 12 Malay \\
\hline \multirow[t]{2}{*}{ Baby gender } & 17 Male & 18 Male \\
\hline & 24 Female & 24 Female \\
\hline $\begin{array}{l}\text { Gestational Age at Delivery } \\
\text { (weeks) }\end{array}$ & $39(37-41)$ & $39(37-41)$ \\
\hline Birth Weight (g) & $2482(2055-2790)^{*}$ & $3133(2955-3540)^{*}$ \\
\hline Birth Weight Percentile & $3.8(0.4-9.6)^{*}$ & $52.1(16.2-85.8)^{*}$ \\
\hline Parity & $1(0-4)$ & $1(0-2)$ \\
\hline Maternal Age (years)a & $29(22-44)$ & $30(20-42)$ \\
\hline \multirow[t]{2}{*}{ Maternal BMIa } & $21.7(17.0-42.3)$ & $23.6(17.5-40.9)$ \\
\hline & $1 / 41 \mathrm{ND}$ & $2 / 42 \mathrm{ND}$ \\
\hline \multirow[t]{2}{*}{ Cigarette Smoking Status ${ }^{b}$} & $1 / 41$ Yes & $0 / 42$ Yes \\
\hline & $0 / 41 \mathrm{ND}$ & $2 / 42 \mathrm{ND}$ \\
\hline \multirow[t]{2}{*}{ Cigarette Smoke Exposureb,c } & $11 / 41$ Yes & $8 / 41$ Yes \\
\hline & $2 / 41 \mathrm{ND}$ & $4 / 41 \mathrm{ND}$ \\
\hline
\end{tabular}

Values with ranges are presented as median; ND - no data available.

* p-value between Cases and Controls below 0.05

a Data from the time of recruitment (first trimester of pregnancy)

b Data from the 26-28 $8^{\text {th }}$ week of pregnancy

c Exposure at home and/or work 
Metabolic profiling using GC-MS revealed over 100 chromatographic peaks (potential metabolites) that were relatively different between cases and controls. Among them, 47 metabolites were identified using an in-house mass-spectrometry library, from which 32 were considered as significantly altered in the FGR cases compared to the controls ( $p<0.01$; Figure 1). The range of these metabolites is given in the supplementary material (Table S1).
Unsupervised multivariate analysis, Principal Component Analysis (PCA), showed a distinctive separation in the metabolic profile of maternal hair between cases and controls (Figure 2A). A multivariate predictive model combining 5 discriminating metabolites (lactate, levulinate, 2-methyloctadecanate, tyrosine, and margarate) produced an area under the receiver-operating curve of 0.998 (95\% CI: 0.992-1.000; Figure 2B).

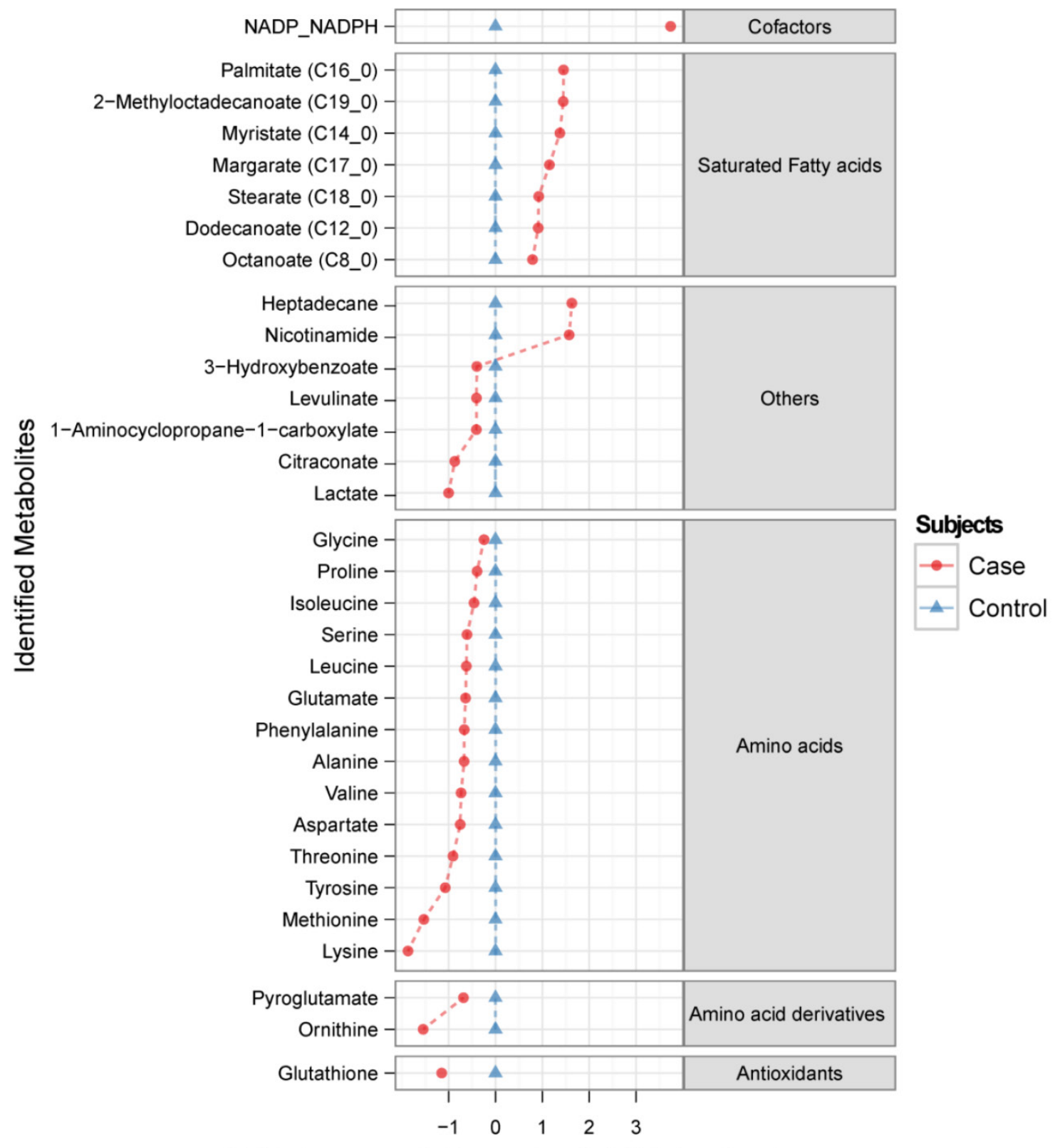

Fold changess: (-)Lower level, (+)Higher level

Figure I. The ratio of identified metabolite levels in maternal hair samples between Fetal Growth Restriction (FGR) case and control groups. Red circles


metabolites generating statistically significant scores $(p<0.01)$ are presented. 

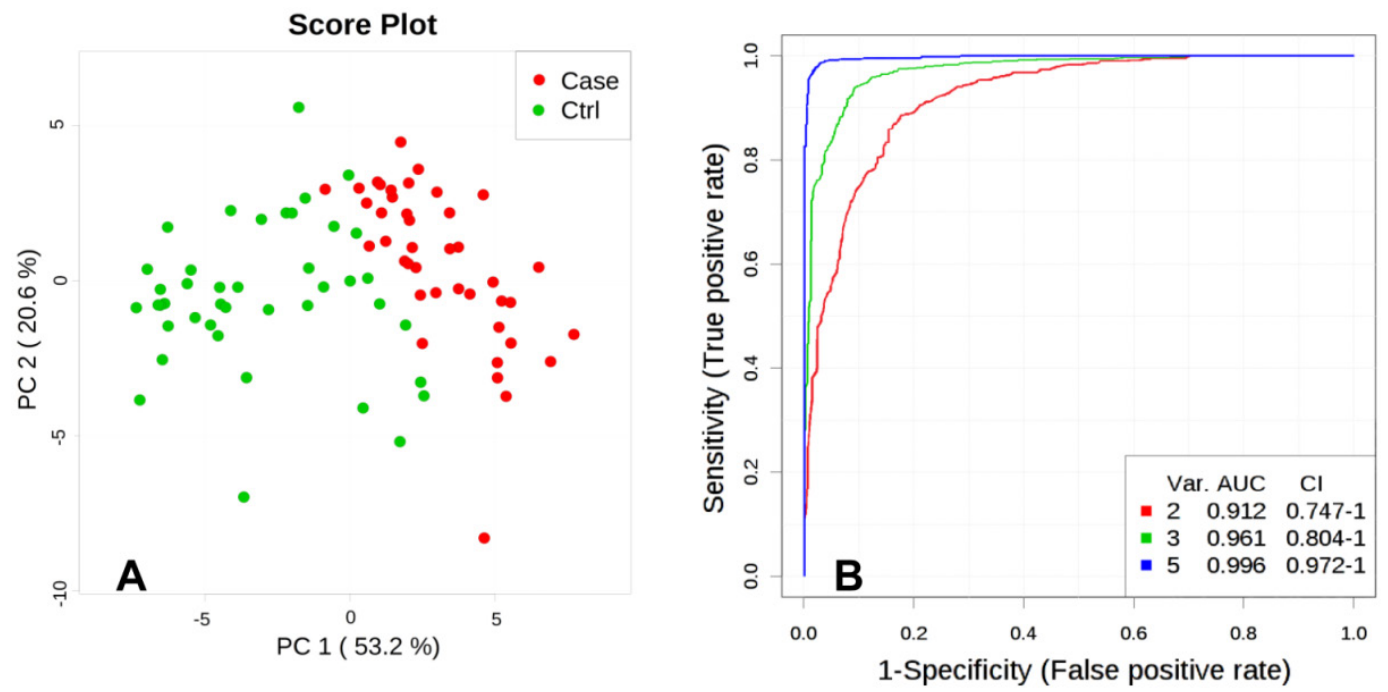

Figure 2. A. Principal Component Analysis (PCA), PCI vs. PC2. Score plot shows separation between Fetal Growth Restriction (FGR) Cases (red) and Controls (green) based on metabolites abundance significantly different $(p<0.0 I)$ between the groups. B. Multivariate ROC curve, based on metabolites which were statistically different between Control and FGR Case groups $(p<0.0 I)$. Var. designated as number of discriminating metabolites $($ lactate, levulinate, 2-methyloctadecanate, tyrosine, margarate; significance to the model decreasing as listed) included in the AUC (Area Under the Curve) calculations.

Biological fluids and tissues are still the most common specimens used in biomarker discovery within metabolomics field ${ }^{23}$. Changes in biochemical profiles of blood and urine can potentially provide information regarding disease development before the full onset of a disease ${ }^{24}$. Discovery of metabolic shifts in biofluids at a very early stage of disease can be highly problematic due to a great number of biochemicals shifting throughout the day, creating a noise. Diet, exercise, sleep pattern and other factors can have a significant effect on the observed metabolome in collected sample. Here we report a new target for metabolic studies, which facilitates a reduction of noise and provides a stable source of chemical information over many months ${ }^{25}$, the metabolomics of hair.

To test this new approach, we analyzed the metabolome of hair from women with pregnancies complicated by FGR when compared to normal controls. The majority of the 32 discriminatory metabolites were amino acids, amino acid derivatives and fatty acids.

Amino acids are important precursors for fetal development and growth, providing the 'building blocks' for the biosynthesis of proteins, nucleotides and neurotransmitters. All 14 of the amino acids measured in our studies were found at reduced concentrations in the hair of women whose pregnancies were complicated by FGR. Interestingly, five of the six most markedly reduced amino acids are considered to be essential amino acids for infants (lysine, methionine, tyrosine, valine and threonine). Essential amino acids are not synthesized by the body and must thus be supplied exogenously (i.e. through the mother's diet or via maternal serum). The mechanisms of amino acid transportation through the hair follicles in relation to FGR is not yet fully understood, although alterations in maternal and fetal plasma amino acids concentrations have previously been reported ${ }^{26-28}$. Fatty acids (from C8 to C19) were the second major class of discriminatory metabolites. In accord with this, we have previously reported decreased fetal and increased maternal plasma fatty acid levels in pregnancies complicated by FGR ${ }^{29}$. This result affirms our hypothesis that much of the composition of hair reflects the time-averaged composition in the circulation.

In addition to the clear differences seen between cases and controls with regard to amino acids and fatty acids we also observed some interesting or unexpected differences for lesser known compounds. The finding of increased heptadecane in the FGR cases is intriguing. Heptadecane, an exogenous alkane hydrocarbon, may represent air pollution and/or food contamination. Heptadecane can be absorbed in the body as well as incorporated into lipids and phospholipids ${ }^{30}$. It is unclear whether heptadecane exposure is an environmental trigger leading to FGR or whether the elevated levels seen in hair are a downstream contaminant arising from another, more important, environmental trigger. Further investigation of this compound and its potentially adverse gestational effects is clearly warranted.

The significantly elevated levels of NADPH/NADP combined with the significantly decreased levels of glutathione in the hair of mothers with FGR babies suggests that there may be general, 
long-term deficits with regard to redox control. Glutathione is an essential antioxidant, acting as a scavenger of reactive oxygen species in mitochondria. NADPH is used by a number of enzymes for generating reduced glutathione, leading to the production of NADP. While our GC-MS method could not distinguish between reduced and oxidized forms of these compounds, the imbalance in their levels certainly suggests that there is a strong coupling between the two. Loss of redox control, oxidative stress and placental oxidative stress has been implicated in a number of perinatal conditions including pre-eclampsia and FGR ${ }^{31}$. These changes are detectable in the serum and placenta of FGR mothers and so it seems reasonable to conclude that these changes could also be reflected in their hair.

Some limitations of our study should be considered. The molecules detected using GC-MS are restricted to volatile molecules and those easily made volatile by derivatization, such as organic acids and amino acids. The MCF derivatization method we used for this study lowers the boiling point of molecules containing $\mathrm{NH}, \mathrm{NH}_{2}$ and $\mathrm{COOH}$ groups. However, our attempts to analyse the hair metabolite profile using LC-MS were restricted by a high concentrations of salts in the extracts after base digestion; these interfered with the LC-MS system and improvements to this method are are prerequisite to effective LC-MS analysis. The low amount of available hair sample neccesitated use of,the full length of hair strands; future analysis should focus on the section of the hair which corresponds to the time of exposure accrual. It should be noted that despite these limitations, complete discrimination of FGR from controls was achieved; this emphasises the robustness of the approach.

\section{Conclusions}

In summary, although the potential for detecting FGR using this methodology is exciting, the wider implications may be even greater; to our knowledge, this is the first description of the metabolomic profile of human hair. It provides interesting insights with regard to the types of endogenous (and potentially exogenous) compounds that are deposited in hair throughout the hair growth cycle. While hair analysis has been performed for many decades, the techniques have always been highly targeted with a specific focus on detecting specific drugs, poisons or toxic metals ${ }^{32}$. As a result, relatively little information has been acquired about other chemicals or common metabolites found in hair and relatively scant attention has been paid to the utility of hair in assessing other kinds of environmental exposures, longer-term physiological changes, nutritional deficits or impaired metabolic processes. Overall, we believe our study highlights the unrealized potential of hair specimen analysis in clinical biomarker discovery and environmental exposure analysis.

\section{Abbreviations}

AUC: Area Under the Curve; FGR: Fetal Growth Restriction; GC-MS: Gas Chromatography - Mass Spectrometry; LC-MS: Liquid Chromatography Mass Spectrometry; GUSTO: Growing Up in Singapore Towards healthy Outcomes, pregnancy cohort; MCF: Methylchloroformate; NADP: Nicotinamide Adenine Dinucleotide Phosphate; PC: Principal Component; PCA: Principal Component Analysis; ROC: Receiver Operating Characteristic; SFH: Symphysis-Fundal Height; SGA: Small for Gestational Age.

\section{Supplementary Material}

Table S1. http:/ / www.thno.org/v04p0953s1.pdf

\section{Acknowledgements}

Authors would like to thank Worldwide Universities Network for the financial support of this project. All metabolome analyses were carried out at the Centre for Genomics, Proteomics and Metabolomics of the University of Auckland. GUSTO is funded by the National Medical Research Council, NMRC/TCR/004-NUS/2008. KS, and PNB are supported by Gravida, National Centre for Growth and Development. PDG is supported by the Singapore Institute for Clinical Sciences. LCK is a Science Foundation Ireland Principal Investigator (08/IN.1/ B2083).

\section{Competing Interests}

The authors have declared that no competing interest exists.

\section{References}

1. Dunn WB, Broadhurst DI, Atherton HJ, Goodacre R, Griffin JL. Systems level studies of mammalian metabolomes: the roles of mass spectrometry and nuclear magnetic resonance spectroscopy. Chemical Society reviews 2011; 40(1): 387-426.

2. Griffin JL, Bollard ME. Metabonomics: its potential as a tool in toxicology for safety assessment and data integration. Current drug metabolism 2004; 5(5): 389-98.

3. Kenny LC, Broadhurst DI, Dunn W, et al. Robust early pregnancy prediction of later preeclampsia using metabolomic biomarkers. Hypertension 2010; 56(4): 741-9.

4. Holmes E, Loo RL, Stamler J, et al. Human metabolic phenotype diversity and its association with diet and blood pressure. Nature 2008; 453(7193): 396-400.

5. Merrick BA, London RE, Bushel PR, Grissom SF, Paules RS. Platforms for biomarker analysis using high-throughput approaches in genomics, transcriptomics, proteomics, metabolomics, and bioinformatics. IARC Sci Publ 2011; (163): 121-42.

6. Dunn WB, Broadhurst D, Begley P, et al. Procedures for large-scale metabolic profiling of serum and plasma using gas chromatography and liquid chromatography coupled to mass spectrometry. Nature protocols 2011; 6(7): 1060-83.

7. Pellis L, van Erk MJ, van Ommen B, et al. Plasma metabolomics and proteomics profiling after a postprandial challenge reveal subtle diet effects on human metabolic status. Metabolomics 2012; 8(2): 347-59. 
8. Psychogios N, Hau DD, Peng J, et al. The human serum metabolome. PloS one 2011; 6(2): e16957.

9. Eastman RR, Jursa TP, Benedetti C, Lucchini RG, Smith DR. Hair as a biomarker of environmental manganese exposure. Environmental science $\mathcal{E}$ technology 2013; 47(3): 1629-37.

10. Thornton JG, Hornbuckle J, Vail A, Spiegelhalter DJ, Levene M. Infant wellbeing at 2 years of age in the Growth Restriction Intervention Trial (GRIT): multicentred randomised controlled trial. Lancet 2004; 364(9433): 513-20.

11. Gardosi J, Kady SM, McGeown P, Francis A, Tonks A. Classification of stillbirth by relevant condition at death (ReCoDe): population based cohort study. BMJ 2005; 331(7525): 1113-7.

12. Bujold E, Roberge S, Lacasse $\mathrm{Y}$, et al. Prevention of preeclampsia and intrauterine growth restriction with aspirin started in early pregnancy: a meta-analysis. Obstet Gynecol 2010; 116(2 Pt 1): 402-14.

13. National Collaborating Centre for Women's and Children's Health. Antenatal Care: Routine care for the healthy pregnant woman. London; 2008.

14. Kean L, Liu D. Antenatal care as a screening tool for the detection of small for dates babies. J Obstet Gynaecol 1995; 16: 77-82.

15. McCowan LM, Harding JE, Stewart AW. Customized birthweight centiles predict SGA pregnancies with perinatal morbidity. BJOG 2005; 112(8): 1026-33.

16. Soh SE, Lee SS, Hoon SW, et al. The methodology of the GUSTO cohort study: a novel approach in studying pediatric allergy. Asia Pac Allergy 2012; 2(2): 144-8.

17. Soh SE, Tint MT, Gluckman PD, et al. Cohort Profile: Growing Up in Singapore Towards healthy Outcomes (GUSTO) birth cohort study. International journal of epidemiology 2013.

18. Smart KF, Aggio RBM, Van Houtte JR, Villas-Bôas SG. Analytical platform for metabolome analysis of microbial cells using methyl chloroformate derivatization followed by gas chromatography-mass spectrometry. Nature Protocols 2010; 5(10): 1709-29.

19. Aggio R, Villas-Boas SG, Ruggiero K. Metab: an $R$ package for high-throughput analysis of metabolomics data generated by GC-MS. Bioinformatics 2011; 27(16): 2316-8.

20. Xia J, Mandal R, Sinelnikov IV, Broadhurst D, Wishart DS. MetaboAnalyst 2.0--a comprehensive server for metabolomic data analysis. Nucleic acids research 2012; 40(Web Server issue): W127-33.

21. Xia J, Broadhurst DI, Wilson M, Wishart DS. Translational biomarker discovery in clinical metabolomics: an introductory tutorial. Metabolomics 2013; 9(2): 280-99.

22. Wickham H. ggplot2: elegant graphics for data analysis. New York: Springer; 2009.

23. Issaq HJ, Veenstra TD. Proteomic and Metabolomic Approaches to Biomarker Discovery Preface. Proteomic and Metabolomic Approaches to Biomarker Discovery 2013;: Xi-Xii.

24. Issaq HJ, Veenstra TD. Biomarker Discovery: Study Design and Execution. Proteomic and Metabolomic Approaches to Biomarker Discovery 2013;: 1-16.

25. Groeneveld MG, Vermeer HJ, Linting M, Noppe G, van Rossum EFC, van IJzendoorn MH. Children's hair cortisol as a biomarker of stress at school entry. Stress 2013; 16(6): 711-5.

26. Cetin I, Alvino G. Intrauterine growth restriction: implications for placental metabolism and transport. A review. Placenta 2009; 30 (Suppl A): S77-82.

27. Cetin I, Ronzoni S, Marconi AM, et al. Maternal concentrations and fetal-maternal concentration differences of plasma amino acids in normal and intrauterine growth-restricted pregnancies. Am J Obstet Gynecol 1996; 174(5): 1575-83.

28. Di Giulio AM, Carelli S, Castoldi RE, Gorio A, Taricco E, Cetin I. Plasma amino acid concentrations throughout normal pregnancy and early stages of intrauterine growth restricted pregnancy. The journal of maternal-fetal \& neonatal medicine : the official journal of the European Association of Perinatal Medicine, the Federation of Asia and Oceania Perinatal Societies, the International Society of Perinatal Obstet 2004; 15(6): 356-62.

29. Horgan RP, Broadhurst DI, Walsh SK, et al. Metabolic profiling uncovers a phenotypic signature of small for gestational age in early pregnancy. Journal of proteome research 2011; 10(8): 3660-73.

30. Tulliez JE, Bories GF. Metabolism of a N-Paraffin, Heptadecane, in Rats. Lipids 1978; 13(2): 110-5.

31. Heazell AE, Moll SJ, Jones CJ, Baker PN, Crocker IP. Formation of syncytial knots is increased by hyperoxia, hypoxia and reactive oxygen species. Placenta 2007; 28 (Suppl A): S33-40.

32. Schramm KW. Hair-biomonitoring of organic pollutants. Chemosphere 2008; 72(8): 1103-11 